\title{
Monte Carlo Simulation Study of a Differential Calorimeter Measuring the Nuclear Heating in Material Testing Reactors
}

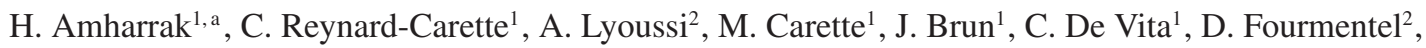 \\ J.-F. Villard ${ }^{2}$, and P. Guimbal ${ }^{3}$ \\ ${ }^{1}$ Aix Marseille Université, CNRS, Université de Toulon, IM2NP UMR 7334, 13397 Marseille, France \\ ${ }^{2}$ CEA, DEN, DER, Instrumentation Sensors and Dosimetry Laboratory, Cadarache, 13108 Saint \\ Paul lez Durance, France \\ ${ }^{3}$ CEA, DEN, DER, SRJH, Cadarache, 13108 Saint Paul lez Durance, France
}

\begin{abstract}
The nuclear heating measurements in Material Testing Reactors (MTRs) are crucial for the study of nuclear materials and fuels under irradiation. The reference measurements of this nuclear heating are especially performed by a differential calorimeter including a graphite sample material. Then these measurements are used for other materials, other geometries, or other experimental conditions in order to predict the nuclear heating and thermal conditions induced in the irradiation devices. This paper will present new simulations with MCNP Monte-Carlo transport code to determine the gamma heating profile inside the calorimeter. The whole complex geometry of the sensor has been considered. We use as an input source in the model, the photon spectra calculated in various positions of CARMEN-1 irradiation program in OSIRIS reactor. After a description of the differential calorimeter device, the MCNP modeling used for the calculations of radial profile of nuclear heating inside the calorimeter elements will be introduced. The obtained results of different simulations will be detailed and discussed in this paper. The charged particle equilibrium inside the calorimeter elements will be studied. Then we will focus on parametric studies of the various components of the calorimeter. The influence of source type will be also took into account. Moreover the influence of the material used for the sample will be described.
\end{abstract}

\section{Introduction}

The nuclear heating (usually expressed in $\mathrm{W}^{-\mathrm{g}^{-1}}$ ) can be described as the rate of energy thermally released in one gram of material subjected to a nuclear radiation flux and corresponds to the increased of the thermal agitation of the electrons inside the irradiated material which leads thus to a temperature increase. In nuclear reactors, the quantity of energy dissipation in a reactor core varies and depends on the location of interaction, the type of interaction, the type of incident radiation and the type of target environment. In materials other than fuel elements, the radiation generated - by particularly gamma photon and neutron particles - deposits its energy in the matter. So the emitted photons and neutrons interact with matter and produce a flux of charged particles that gradually decrease until thermal equilibrium is reached in matter. Nuclear heating is a great deal of interest at the moment as the

\footnotetext{
${ }^{a}$ Corresponding author: hicham. amharrak@univ-amu.fr
}

This is an Open Access article distributed under the terms of the Creative Commons Attribution License 2.0, which permits unrestricted use, distribution, and reproduction in any medium, provided the original work is properly cited. 
measurement of such heating is an important issue for Material Testing Reactors (MTRs). This need is especially generated by the new Jules Horowitz Reactor (JHR), under construction at CEA/Cadarache "French Alternative Energies and Atomic Energy Commission", for which the knowledge of nuclear heating is necessary to size accurately the thermally and mechanical devices hosting irradiation samples. Indeed the expected nuclear heating rate is about $20 \mathrm{~W} \cdot \mathrm{g}^{-1}$ for nominal capacity of $100 \mathrm{MW}$ [1], against currently $13 \mathrm{~W} \cdot \mathrm{g}^{-1}$ in the irradiation OSIRIS reactor at CEA-Saclay [2]. This level of nuclear heating rate should be compared with measurements performed in critical mock-up reactors of very low power, such as MINERVE and EOLE at the CEA/Cadarache, where nuclear heating reach a value from $10^{-7}$ to $10^{-6} \mathrm{~W} \cdot \mathrm{g}^{-1}[3,4]$.

To improve the qualification of the nuclear heating inside experimental channels of the JHR reactor, a research program between CEA and the University of Aix-Marseille, called IN-CORE "Instrumentation for Nuclear radiation and Calorimetry On line in REactor", started in 2009. This program has a dual objective. The first one focuses on the development of an innovative technological device mixing different types of sensors as fission and ionization chambers to measure neutron and photon fluxes, respectively, as well as a gamma thermometer and a radiometric calorimeter to qualify nuclear heating. The second objective is to optimize the measurement method to quantify nuclear heating in these experiments: Radiometric calorimeter (the sensor and the calibration method). The first prototype was already developed and two irradiation campaigns were performed successfully in the CEA MTR OSIRIS in 2012 for nuclear heating levels up to $2 \mathrm{~W} \cdot \mathrm{g}^{-1}[1,5]$. This prototype includes two mock-ups dedicated respectively to photonic measurements (CARMEN-1P) or neutronic measurements (CARMEN-1N) and contains a specific differential calorimeter [6]. The preliminary analysis shows that the first calculations (reduced to the graphite sample of the calorimeter) overestimates the measurements by about 20\% [1]. The neutron and photon spectra were calculated inside 4 water channels located in the OSIRIS water reflector with the Monte Carlo code TRIPOLI4® [7] and the European JEFF3.1.1 nuclear data library [8]. They correspond to the different positions of CARMEN-1 irradiation program.

Nuclear heating is often estimated by the quantity KERMA (Kinetic Energy Released per MAss unit) defined as the sum of the initial kinetic energies of all the charged particles liberated by uncharged ionizing radiation. KERMA calculation requires a Monte Carlo calculation in neutron mode (neutron heating) or neutron-photon mode (photon heating) $[9,10]$. However, this quantity is representative of the heating only when the charged particle equilibrium is reached and in the case of a system made of a single material. Therefore, a another approach to determine this estimation is made in this study by considering another physical parameter which is more representative of heating: the mass energy deposition by the generated charged particles. The determination of this quantity then requires a more comprehensive neutron-photon-electron mode calculation [3].

This paper focuses on the estimation of the heating due to the prompt gammas by these two quantities (energy deposition and KERMA). These quantities are calculated by Monte Carlo simulations using the transport code MCNP "Monte Carlo N-Particle" [11]. They will be considered in the case of the differential calorimeter of CARMEN-1P device which was irradiated in the reflector of OSIRIS reactor. Thus after having detailed the model of the complete geometry of the sensor, the impact of the thickness and the type of material surrounding the calorimeter sample will be determined. Then, the influence of the nature of the sample will be considered for different types of source. The results of these two values will be compared to those obtained for the reference graphite sample. Finally, these results will be discussed and one example of sensor optimization will be given.

\section{Modeling of Nuclear Heating in MTR Reactors}

This paragraph is dedicated to the modeling of gamma heating in the differential calorimeter of CARMEN-1P device, which was irradiated in the reflector of the OSIRIS reactor. This modeling is performed using the MCNP transport code. 


\subsection{Origin of the Nuclear Heating}

During the operation of a nuclear reactor, a number of particles are generated: neutrons, photons $(\gamma, \mathrm{X} .$.$) , light charged particles (electron and positron), heavy charged particles (fission fragments,$ proton, alpha...). Most of the energy deposition in nuclear fuel results from the total absorption of the kinetic recoil energy from fission products by the atoms and molecules forming the medium. Apart from the fuel elements, the radiation generated - particularly photons, neutrons and light charged particles (beta- deposits its energy in the matter. The emitted photons and neutrons interact with the material and produce a flux of charged particles that gradually decrease until thermal equilibrium is reached in the material. The successive energy deposits create local heating that will result in a temperature increase in the material.

Outside the fuel, nuclear heating of materials has three components:

- Prompt photons (fission, capture and scattering), which represents the major component. This heating comes primarily from energy deposition during photon-matter interactions by Photoelectric effect, Compton scattering, and Pair production.

- Delayed photons, emitted by radioactive decay of fission products;

- Neutrons, which are minority. They come from the following reactions: Elastic and Inelastic Scattering of Neutrons, Beta decay, $(\mathrm{n}, \gamma)$ or $(\mathrm{n}, \mathrm{p})$ reactions.

So a part of the energy carried by photons is transferred via interactions to charged particles. The transmitted energy per mass unit of all charged particles, in the form of kinetic energy, corresponds to the quantity KERMA. Moreover, the generated electrons deposit their energy in the medium: this energy deposition per mass unit corresponds to the absorbed dose. The photon KERMA, expressed in $\mathrm{MeV} / \mathrm{g}$, is given by:

$$
K=\int_{E_{\gamma}} \phi\left(E_{\gamma}\right) \cdot\left(\frac{\mu_{t r}}{\rho}\right) \cdot E_{\gamma} \cdot d E_{\gamma}
$$

Where: $\phi$ is the photon fluence $\left(\mathrm{cm}^{-2}\right), E_{\gamma}$ is the photon energy $(\mathrm{MeV})$ and $\left(\mu_{t r} / \rho\right)$ is the mass energytransfer coefficient of photons $\left(\mathrm{cm}^{2} \cdot \mathrm{g}^{-1}\right)$.

When charged-particle equilibrium is reached in the medium, a relationship between KERMA and energy deposition exists:

$$
D=K \cdot(1-g) .
$$

Where $\mathrm{g}$ represents the bremsstrahlung fraction.

\subsection{Differential Calorimeter Presentation}

The differential calorimeter is made of two superposed twin cells surrounded by a stainless steel jacket: a sample calorimetric cell and a reference calorimetric cell. The body of each cell is divided into three main parts (cf. Fig. 1): a head, a rod and a base in contact with the jacket [6]. The first upper cell, called sample cell, contains a graphite sample inside its head. The second lower cell, called reference cell, is reduced to the body (the sample is replaced by nitrogen).

This reference cell is used to determine the nuclear heating of the body and to avoid its influence on the sample cell response which is due to a total nuclear heating deposition on the cell body and on the sample. More precisely, the nuclear heating deposition on the graphite sample is obtained from two temperature difference measurements obtained respectively on each instrumented cell by two thermocouples (type K) (located under the cell head (black) and on the middle height of the cell base (white)). 


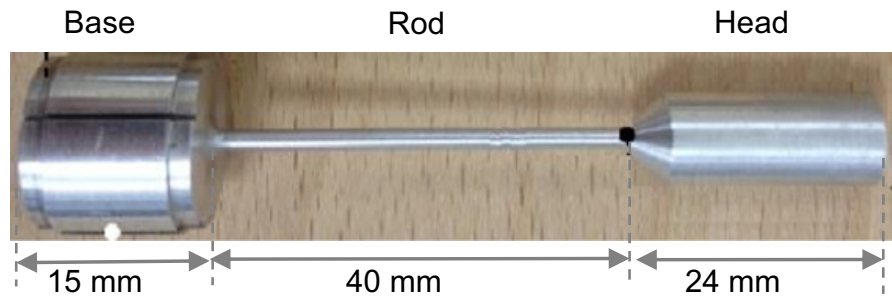

Figure 1. Calorimeter cell of CARMEN-1P device.

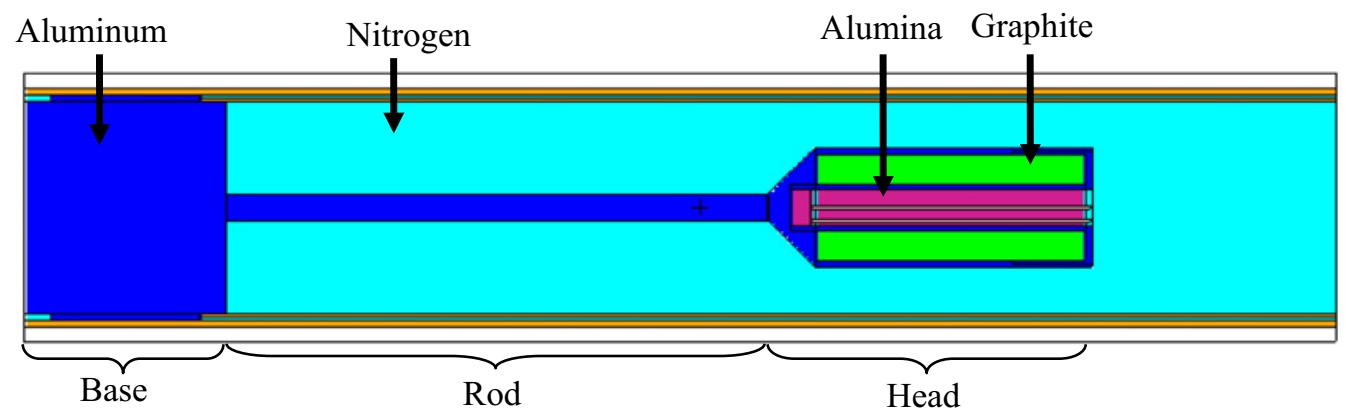

Figure 2. Axial cross section of MCNP modeling of a calorimeter sample cell.

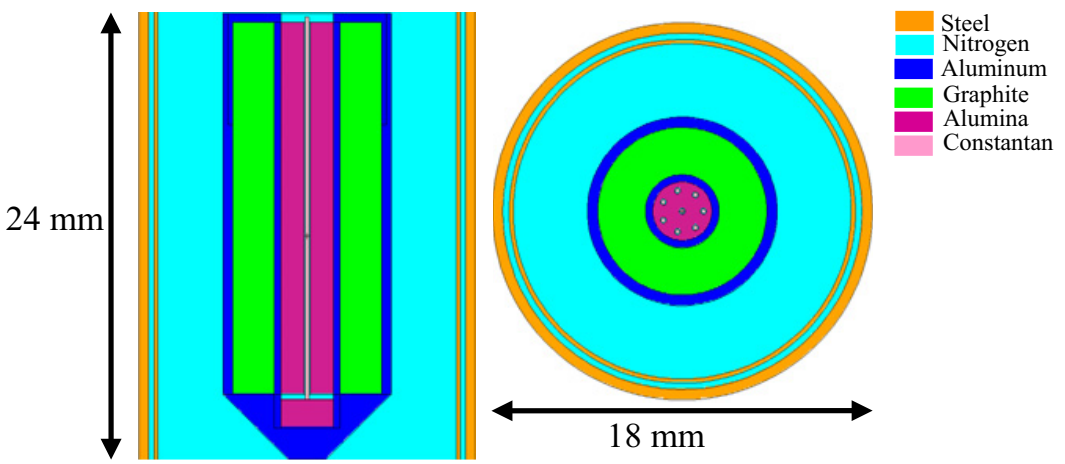

Figure 3. Radial cross section of MCNP modeling of calorimeter cell head.

\subsection{MCNP Differential Calorimeter Model}

The cell of the differential calorimeter is modeled using the MCNP code. This section presents the geometric model considered for the sample cell and associated physical modeling. The geometry of the model is described in the code input file, in two steps. In a first step we define the surfaces and their associated axes. In a second-step, we construct the geometry cell by cell, from unions, intersections or exclusions of these surfaces. The model includes the complete geometry of the sample cell and the surrounded jacket. The geometry of the model is described in the Fig. 2 and Fig. 3 [12]

Physical modeling allows to associate to each cell a material and nuclear data libraries (photonmatter interaction cross-sections). The characteristics of the particle source are then described in terms of its nature, position, intensity, energy distribution and its emission direction. Finally, it is necessary to define the type of output results called "Tally" calculated by the code. Indeed, the code allows to 


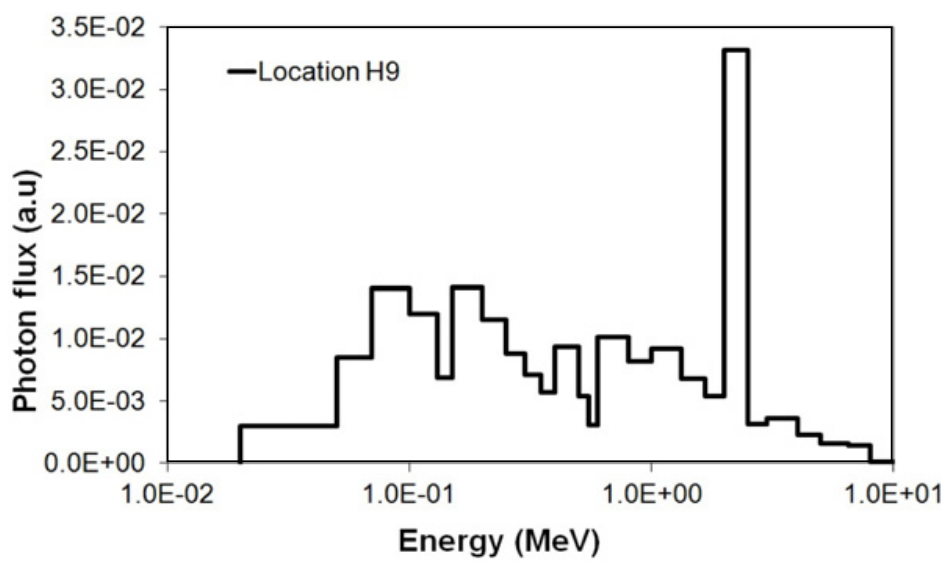

Figure 4. Photon spectrum inside the water channel located into the OSIRIS reflector.

evaluate parameters such as current, flux, energy deposition, at different points, surfaces or volumes. The output results of the code are systematically normalized by particle source (/p-src). To evaluate the KERMA and energy deposition from a photon spectrum as input, the photon-electron MCNP simulation is necessary. The simulations have a number of assumptions and limitations, such as cut off energy or the fact that, only the modeled area of interest contributes to energy deposition in the sample.

The photon (fission, capture and scattering) spectrum (cf. Fig. 4) are calculated inside the water channel H9 located in the OSIRIS water reflector with the Monte Carlo code TRIPOLI4® [7] and the European JEFF3.1.1 nuclear data library. This prompt photon spectrum does not take into account the delayed photons and the photons from activation. The spectrum was used as input in the source of the MCNP model.

\section{Parametric Studies on the Energy Deposition in the Calorimeter}

Simulation results presented in this paragraph concern a parametric study of the prompt photon heating in the case of a cylindrical source at the location H9. The prompt photon heating in the sample was estimated by the two quantities: energy deposition and KERMA (in these studies, expressed in $\mathrm{MeV} / \mathrm{g}$ by particle source). The influence of various parameters, such as the thickness and nature of the jacket, the nature of the sample and structure materials and the type of the photon source is determined on these two quantities.

\subsection{Influence of the Thickness of the Steel Jacket}

Photon KERMA depends of the level flux, the energy of photon and the mass energy-transfer coefficient (Eq. (1)). However the energy deposition per mass unit depends of mass stopping power of electrons generated in the surrounding. Thus it is important to take into account the complete geometry of the calorimeter in the model, with the surrounding materials.

We studied the influence of the thickness of the steel jacket on KERMA and on the energy deposition in graphite. The obtained simulation results are plotted in graphite (Fig. 5). The obtained results in various areas of the calorimeter (base, rod and head) are normalized by the maximum value (Fig. 6).

The photon attenuation induced by the increase of the steel thickness, leads to a decrease of the KERMA in graphite (Fig. 5). In the case of small thickness of the jacket, the head of the calorimeter 


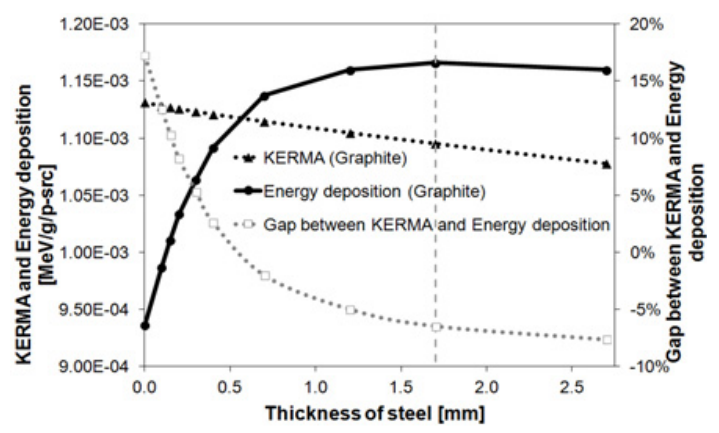

Figure 5. Comparison between KERMA and energy deposition.

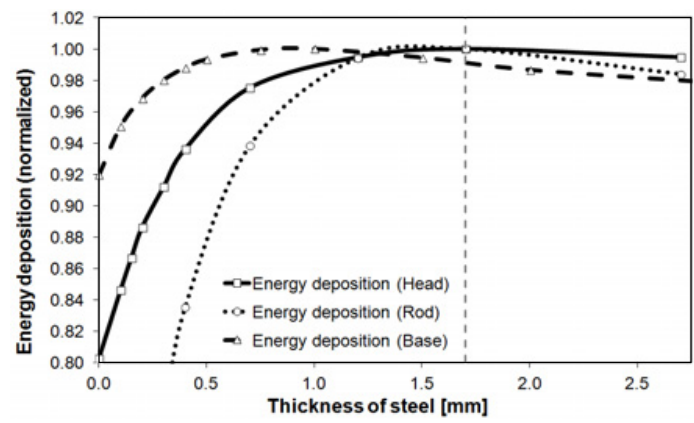

Figure 6. Variation of the energy deposition in the head, rod and base.

would be surrounded only by nitrogen and the energy deposition in the graphite would decrease considerably. According to this study, optimal electronic equilibrium conditions can be reached for a $1.7 \mathrm{~mm}$ thickness of steel jacket. This thickness is sufficient to reach electronic equilibrium in the rod and in the base of the calorimeter (see Fig. 6). Therefore, the jacket thickness of the present configuration equal to $0.7 \mathrm{~mm}(0.5 \mathrm{~mm}$ of jacket and $0.2 \mathrm{~mm}$ of stainless steel brace close fitting to the jacket) does not allow a good electronic equilibrium. This underestimated an error of $2.5 \%$ on the energy deposition.

We studied the influence of the steel jacket thickness on the radial profile of the gamma flux, the KERMA and the energy deposition. The obtained simulation results (at mid-height of the head) are plotted in Figs. 7 and 8.

From these simulation results, the influence of the steel jacket on the radial profile of KERMA is less important (about 2\%) than the influence on the radial profile of the energy deposition (about 18\%). These results confirmed the need to estimate the gamma heating by using the energy deposition.

\subsection{Influence of the Nature of the Materials}

\subsubsection{Influence of the Nature of the Jacket Material}

To study the influence of the nature of the jacket material on the energy deposition and KERMA in the sample of graphite, we tested other materials such as graphite, aluminum, zirconium and bismuth.

We observe on Fig. 9 that when the density of the jacket material increases, the thickness necessary to achieve the electronic equilibrium decreases. This thickness corresponds to the mean free path of electrons in each type of material. According to this study, a thickness of $0.7 \mathrm{~mm}$ is sufficient to achieve electronic equilibrium for Bismuth, against $1.7 \mathrm{~mm}$ in the case of steel or zirconium. A thickness of 


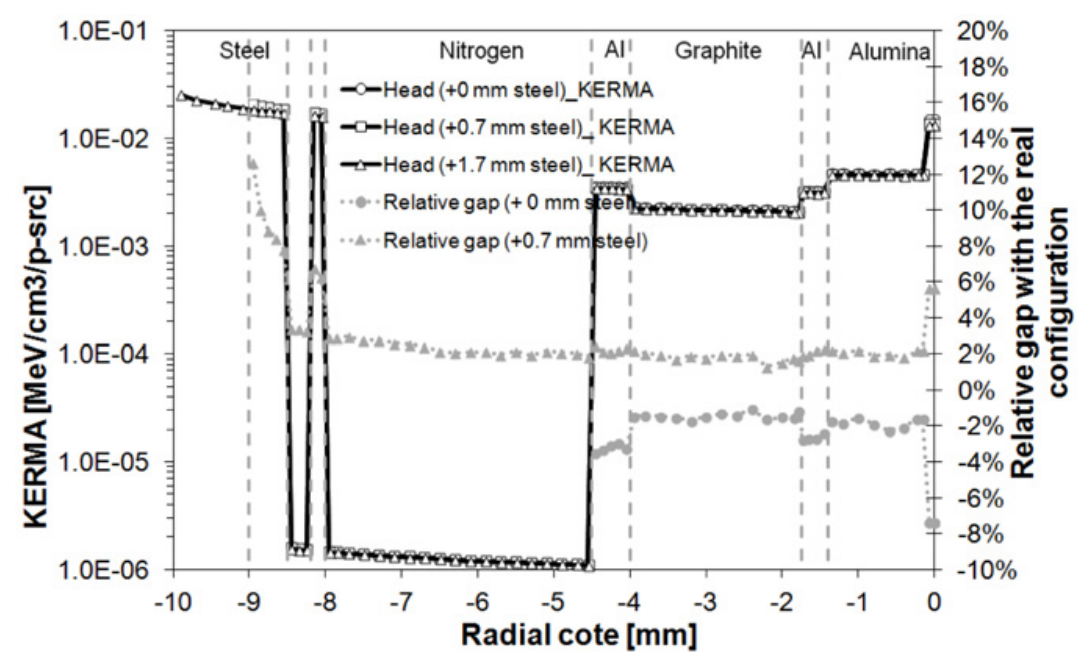

Figure 7. Radial profile of KERMA for various thicknesses of jacket made of steel.

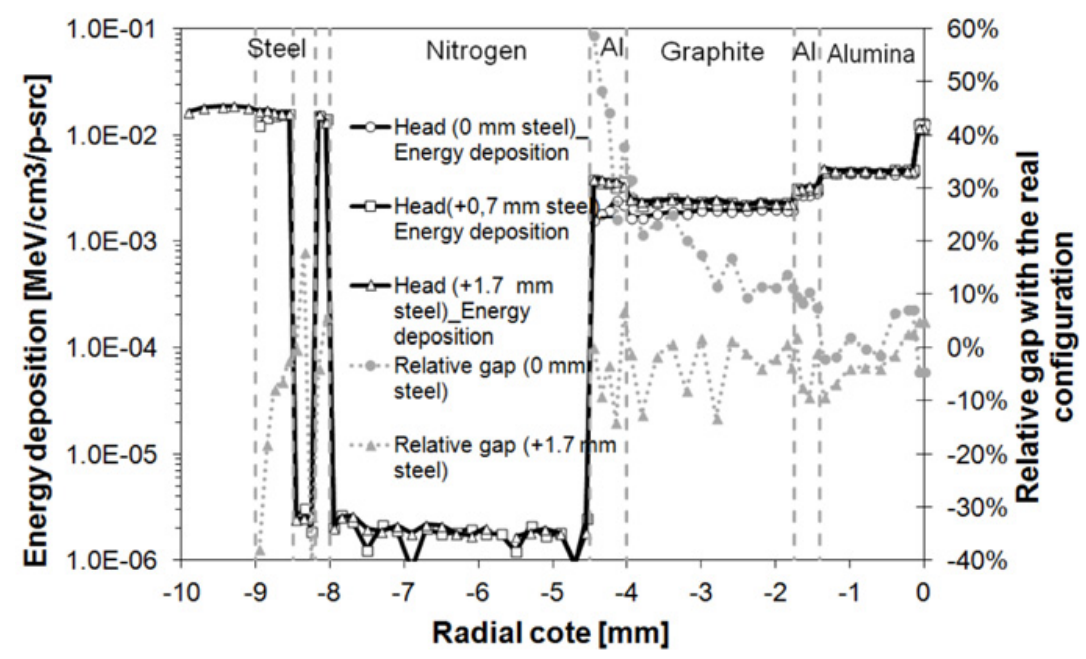

Figure 8. Radial profile of the energy deposition for various thicknesses of jacket made of steel.

$3.5 \mathrm{~mm}$ is needed in the case of aluminum and graphite. In the case of Bismuth, for a thickness greater than $0.7 \mathrm{~mm}$, the decrease of energy deposition versus the thickness of the jacket is more important than for the other materials, this is due to the photons attenuation induced by the increase of the Bismuth thickness.

The Fig. 10 shows the evolution of the KERMA and energy deposition in the graphite sample versus the atomic number of the jacket material in the case of a jacket thickness equal to $0.7 \mathrm{~mm}$ (current configuration). Thus induces an important difference in the energy deposition inside the graphite sample. In particular we can observe for two materials most commonly used in jacket designing that the energy deposition in the case of steel jacket is $14.7 \%$ greater than in the case of aluminum jacket. 


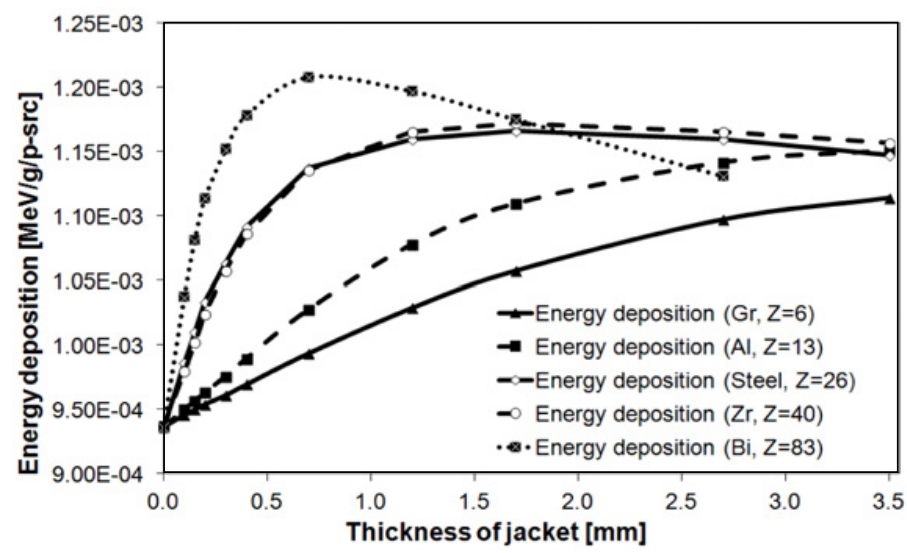

Figure 9. Energy deposition versus jacket thickness for various materials.

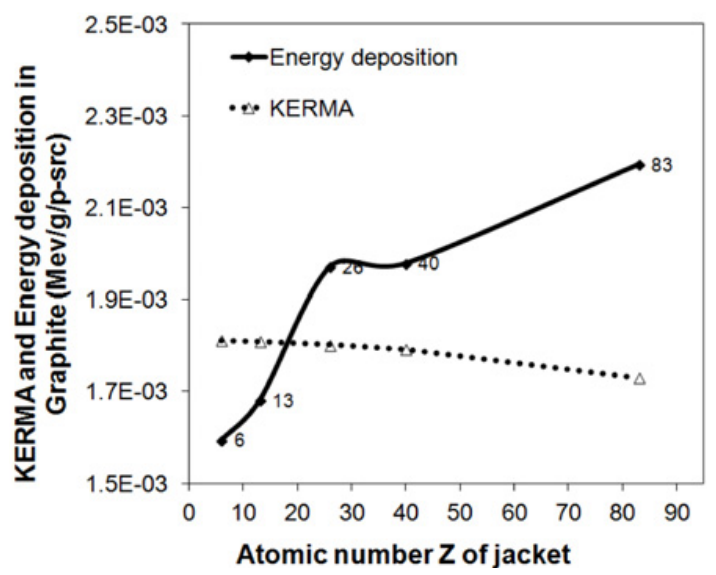

Figure 10. Evolution of KERMA and energy deposition versus the atomic number of jacket.

\subsubsection{Influence of the Nature of the Calorimeter Materials (Structure and Sample)}

The influence of the material used for the head of the calorimeter on KERMA and energy deposition in sample of graphite is illustrated in Figure 11. It is found that the attenuation of gamma flux through the different materials leads to a decrease of the KERMA versus atomic number $\mathrm{Z}$.

The influence of the nature of the structure material on energy deposition in graphite is lower that the influence of the jacket material. We observe a difference equal of $3 \%$ between an aluminum and a steel structure. However in the case of a high heating level, an aluminum structure is more suitable due to its low density resulting in a lower deposition and lower temperature.

Finally the influence of the nature of the sample was studied. Figure 12 illustrates the evolution of the KERMA and energy deposition versus the atomic number of the sample in the case of two types of photon sources (cylindrical or punctual).

From these results, it appears that when $\mathrm{Z}$ increases, the gap between the KERMA and the energy deposition increases whatever the type of the source. So to measure a low heating level, a sample with a high atomic number would be chosen to increase the sensitivity of the sensor. 


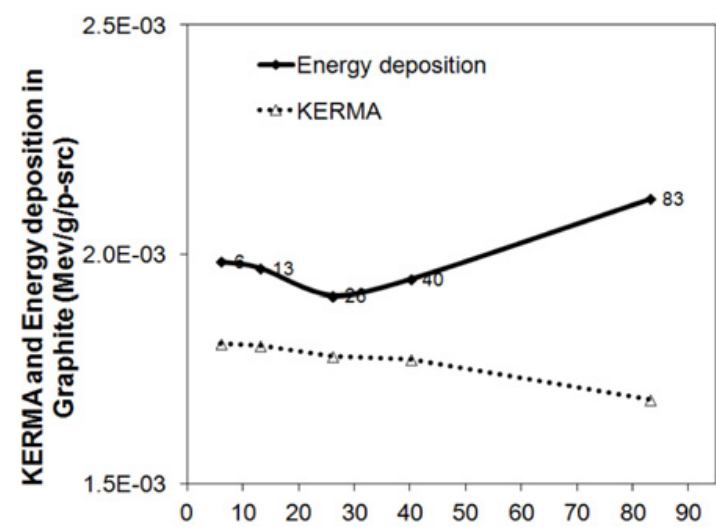

Atomic number $\mathbf{Z}$ of structure material

Figure 11. Evolution of KERMA and energy deposition versus the atomic number of the structure material.

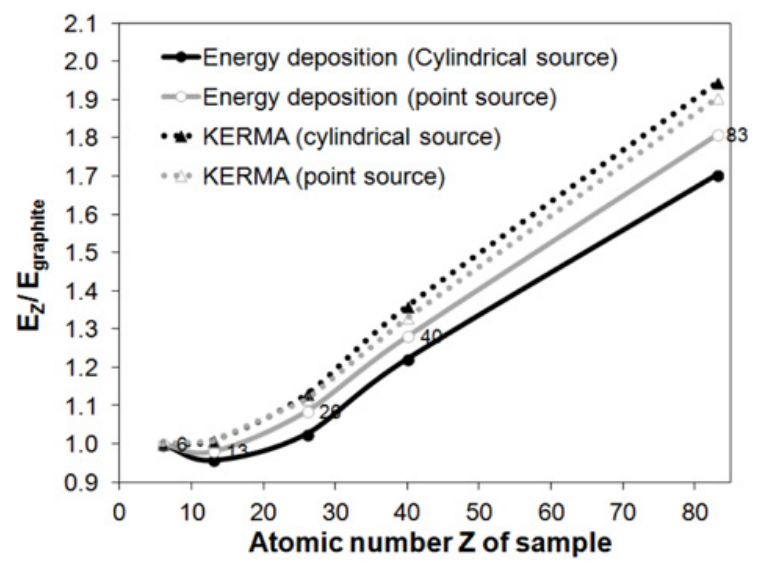

Figure 12. Evolution of the KERMA and the energy deposition versus the atomic number of the sample.

\section{Conclusion and Perspectives}

A parametric study of the KERMA and the energy deposition in the sample of the calorimetric cell of the CARMEN type calorimeter has been performed. There was a significant difference between the values of the gamma heating estimated by these two quantities (KERMA and energy deposition). Thus the gamma heating is better estimated by the energy deposition. The influence of the jacket of the calorimeter cell (dimension and nature) is more important than the influence of the cell structure (nature). The influence of the nature of the sample is significant too. These results will be used for the optimization of new calorimeter designs.

This work will be completed in a near future by considering the neutron heating part and the delayed gamma heating.

We acknowledge FEDER, Conseil Régional PACA and Ville de Marseille to support the IN-CORE program. 


\section{References}

[1] D. Fourmentel, C. Reynard-Carette, A. Lyoussi, J. F. Villard, J. Y. Malo, M. Carette, J. Brun, P. Guimbal, and Y. Zerega, IEEE Trans. Nucl. Sci., 60(1), Part: 2, pp. 328-335 (2013)

[2] H. Carcreff, V. Cloute Cazalaa, L. Salmon, IEEE Trans. Nucl. Sci., 59(4), pp. 1369-1376 (2012)

[3] H. Amharrak, J. Di Salvo, A. Lyoussi, A. Roche, M. Masson-Fauchier, A. Pepino, J. C. Bosq, and M. Carette, IEEE Trans. Nucl. Sci., 59(4), pp. 1360-1368 (2012)

[4] H. Amharrak, J. Di Salvo, A. Lyoussi, M. Carette, C. Reynard Carette, Nuclear Inst. and Methods in Physics Research A, Vol. 749, pp. 57-67 (2014)

[5] D. Fourmentel, P. Filliatre, J.-F. Villard, A. Lyoussi, C. Reynard-Carette, H. Carcreff, Nuclear Inst. and Methods in Physics Research A, 724, pp. 76-82 (2013)

[6] J. Brun, C. Reynard-Carette, A. Lyoussi, O. Merroun, M. Carette, A. Janulyte, Y. Zerega, J. Andre, G. Bignan, J-P.Chauvin, D. Fourmentel, C. Gonnier, P. Guimbal, J-Y. Malo and J-F. Villard, IEEE Trans. Nucl. Sci., Volume: 59(6), Part: 2, pp. 3173-3179 (2012)

[7] O. Petit, F. X. Hugot, Y. K. Lee, C. Jouanne, A. Mazzolo, Report CEA-R-6169, Code available from OECD/NEA Data Bank (2008)

[8] A. Santamarina, D. Bernard, P. Blaise, M. Coste, A. Courcelle, and T. D. Huynh, NEA, JEFF Report 22 (2009)

[9] F. Malouch, Journal of ASTM International, 9(4), pp. 423-436 (2012)

[10] A. Péron, F. Malouch, C. M. Diop, In Proc. Int. Conf. ANIMMA’2013, June 23-27, Marseille (France) (2013)

[11] MCNP5 Manual, X-5 Monte Carlo Team, Los Alamos National Laboratory (2005)

[12] H. Amharrak, C. Reynard-Carette, A. Lyoussi, M. Carette, D. Fourmentel, J. Brun, C. De Vita, J-F. Villard, P. Guimbal, Congrès français de thermique 2014, du 3 au 6 juin 2014, Lyon (France) 